\title{
Latest trends in drugs of abuse - HIV infection and neuroAIDS
}

Sneham Tiwari', Madhavan PN Nair ${ }^{2}$ \& Shailendra K Saxena*1

'CSIR-Centre for Cellular \& Molecular Biology, Uppal Road, Hyderabad 500007 AP, India ${ }^{2}$ College of Medicine, Florida International University, Miami, FL 33799, USA

*Author for correspondence: Tel.: +91 4027192630 (Direct); 27160222 - 41 Ext. 2630 = Fax: +91 4027160591 ;

+914027160311.m shailen@ccmb.res.in; shailen1@gmail.com

\section{Symposium on Drugs of Abuse - HIV Infection and NeuroAIDS: A Global Perspective, during the 3rd World Congress on Biotechnology} Hyderabad, India, 13-15 September 2012

Drug abuse and co-occurring infections are associated with significant morbidity and mortality. In particular, HIV infection is associated with serious neurological complications, including neuroAIDS. Therefore, on 13-15 September 2012, the OMICS Group (USA) and Shailendra K Saxena (Centre for Cellular and Molecular Biology, India) hosted a symposium titled: 'Drugs of Abuse - HIV Infection and NeuroAIDS: A Global Perspective' that was cochaired by Jag H Khalsa and Jeymohan Joseph of the NIH, MD, USA, at the 3rd World Congress on Biotechnology, in Hyderabad, India. Renowned scientists from India and the USA highlighted a number of issues, including the epidemiology, causes and underlying pathophysiological mechanisms of neuroAIDS, impact on health, and designing new treatment modalities (e.g., nanotherapeutics) for the treatment of neurological disorders.

Global prevalence of drug abuse indicates that 153-300 million people regularly use illicit drugs (cocaine, opiates, amphetamines and cannabis) [101]. Drug abuse is associated with serious widespread medical and health consequences, such as neurotoxicity (cocaine and methamphetamine [METH]), impairment of the immune system function and many other physiological effects. Alongside this, an estimated 34 million people are infected with HIV [1]. HIV-1 is a retrovirus that infects $\mathrm{CD}^{+}{ }^{+}$T-cell lymphocytes and macrophages, causing profound immunosuppression that may eventually develop into full-blown AIDS. The virus affects almost every physiological system, including the brain, leading to a myriad of neurological complications, including neuroAIDS. Therefore, Jag $\mathrm{H}$ Khalsa (Chief, Medical Consequences of Drug Abuse and Co-occurring Infections, US National Institute on Drug Abuse, NIH, MD, USA) and Jeymohan Joseph (Chief, HIV Neuropathogenesis, Genetics and Therapeutics Branch, Division of AIDS Research, US National Institute of Mental Health, NIH) were invited to cochair a symposium on Drugs of Abuse - HIV Infection and NeuroAIDS: A Global Perspective, at the 3rd World Congress on Biotechnology, September 13-15 2012, in Hyderabad, India (FIGURe 1).

\section{Overview of NIMH-funded research on neuroAIDS}

Jeymohan Joseph provided an overview of the NeuroAIDS research programs and priorities supported by the 'HIV Neuropathogenesis, Genetics and Therapeutics' Branch, Division of AIDS Research at the US National Institute of Mental Health. This Branch supports an integrated program of studies to investigate the pathophysiology and genetic factors that contribute to HIV-associated neurologic and neurocognitive dysfunction (HAND). Support is also provided for developing novel therapeutic strategies to mitigate the CNS complications of HIV infection, using information gathered from research into HIV neuropathogenesis.

There are three major programs within the 'HIV Neuropathogenesis, Genetics and Therapeutics' branch. The 'Mechanisms of HIV Neuropathogenesis' program supports basic research into the mechanisms of HIV-associated neuropathogenesis and associated infections in developed and developing countries. It encourages research into HIV neuropathogenesis that makes use of state-of-the-art approaches from such diverse fields as molecular biology, physiology, virology, neurology, immunology, neuropsychology and epidemiology. The 'Viral and Host Genetics' program supports both basic and clinical research that attempts to determine the viral and host genetic
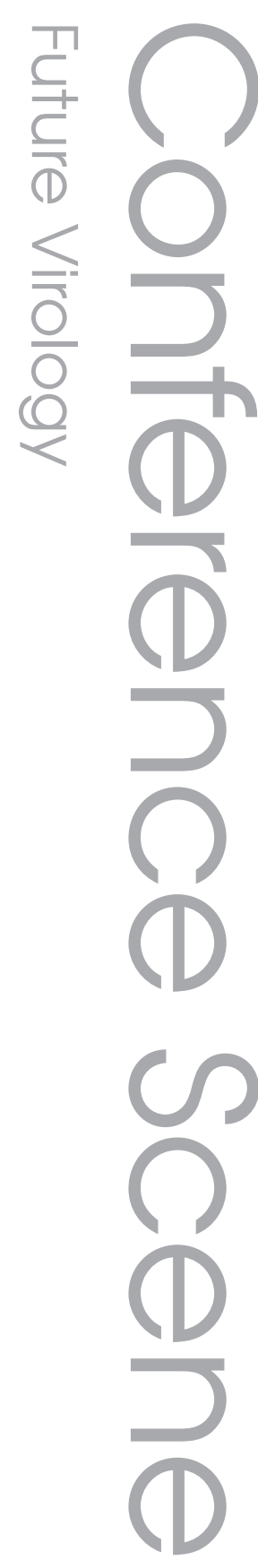
factors that regulate HIV-associated neurologic and neurocognitive complication development in developed and developing countries. It encourages researchers to make use of state-of-the-art genetic approaches (such as transcriptomics, phenomics, epigenomics, whole-genome association studies, next-generation sequencing, exome sequencing and systems biology) to identify and validate the factors that influence HAND pathophysiology. The 'HIV NeuroAIDS Therapeutics' program supports bench-to-bedside research with the aim of encouraging the development of new therapeutic options for HAND. It supports: preclinical-toclinical research on both existing and novel drug targets; the development of novel drug-delivery techniques and approaches, including methods for transporting antiretroviral drugs into the CNS; and creative and original research with the aim of translating novel treatments and therapeutic strategies to a clinical setting. Additionally, basic and clinical studies into HAART-related neurotoxicity are encouraged.

Some of the key priority areas within these programs include:

- Study of the impact of aging and associated co-morbidities in the pathophysiology of $\mathrm{HIV}$-associated CNS disease;

- Understanding the role of global clade diversity and host genetic factors in HIV neuropathogenesis;
- Developing novel therapeutics emerging from HIV neuropathogenesis research;

- Exploring novel approaches for eradication of HIV/CNS reservoirs;

- Moving novel NeuroAIDS therapies to the clinic.

Many of the key program areas of interest are outlined in the Funding Opportunity Announcement, 'HIV Infection of the Central Nervous System (PA-11-014)' [102]. Joseph also described the global NeuroAIDS efforts supported by the National Institute of Mental Health. These include studies supported in India, China, Thailand, Romania, Brazil, South Africa, Nigeria, Zambia, Uganda and Senegal. Preliminary studies emerging from these countries suggest that HIV-1 caused neurocognitive impairments in all countries studied. However, HIV-1 clade D was found to cause a greater incidence of HIV-associated dementia compared with clade A in Uganda. Additional studies are ongoing to further define the impact of HIV clade diversity on neuropathogenic mechanisms from a global perspective.

\section{NeuroAIDS \& differential effects of clades}

The molecular mechanisms behind differential neuroAIDS by HIV-1B and $-1 \mathrm{C}$ clades were explained by Shailendra K Saxena of the Centre

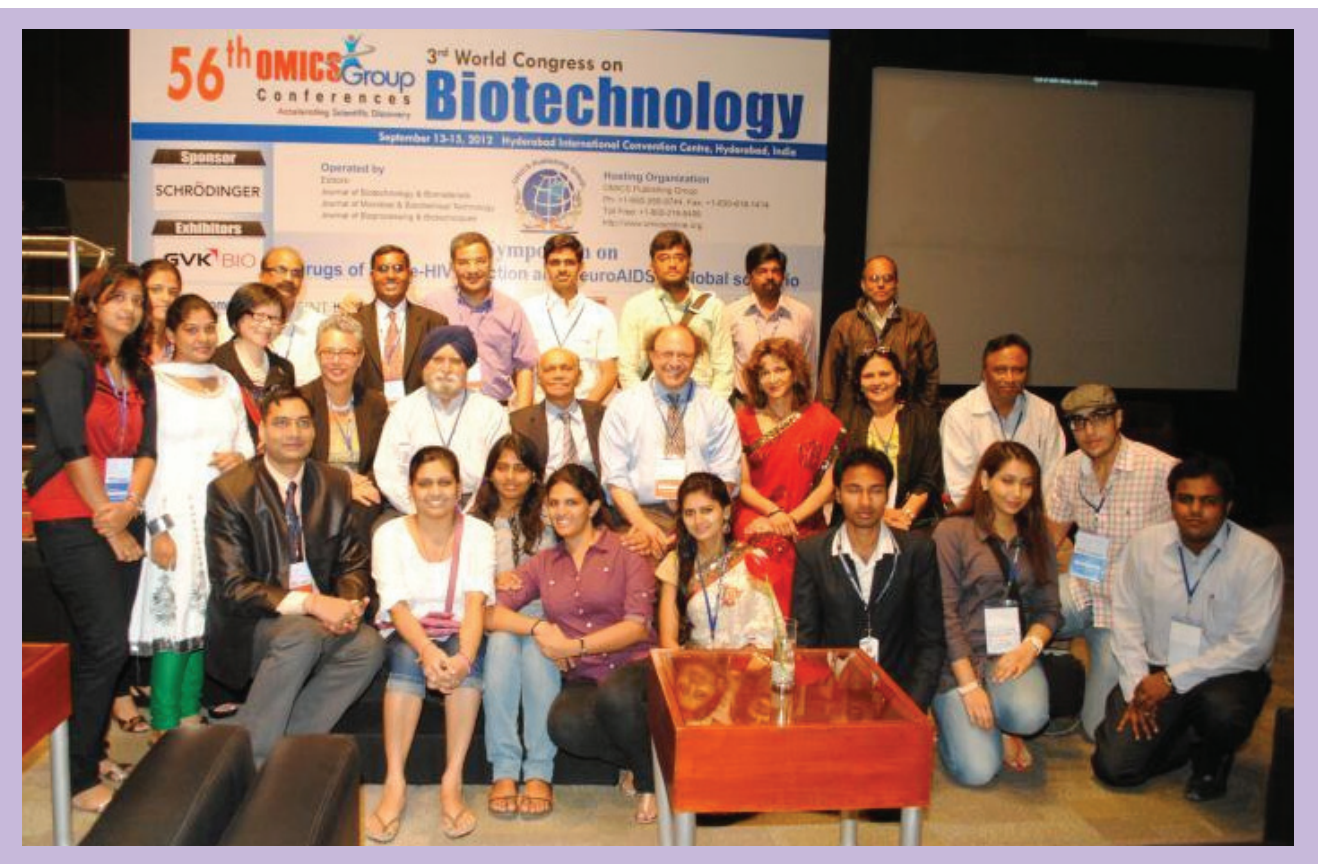

Figure 1. Eminent speakers and delegates during the Symposium on Drugs of Abuse - HIV Infection and NeuroAIDS: A Global Perspective, during the 3rd World Congress on Biotechnology at Hyderabad International Convention Centre, Hyderabad, India (2012). 
for Cellular and Molecular Biology, India (coauthored by Madhavan PN Nair and Thangavel Samikkannu), convener of the symposium. Saxena described that there is an association between HIV, immune dysfunction and APC suppression, factors that lead to damaged neuronal functions. The neurotoxic factor kynurenine, rate-limiting enzyme IDO, 5-HT, and 5-HT transporter (5-HTT) play roles in tryptophan deficiency, leading to serotonergic dysfunction. HIV-1 Tat protein plays an important role in immune dysfunction and, as Saxena explained, may be responsible for the differential manifestations of neuronal dysfunctions in HIV-1B and -1C. According to Saxena, HIV-1B Tat upregulates IDO and downregulates the 5-HTT gene, causing 5-HT reduction with simultaneous increase in kynurenine levels, as compared with HIV-1C Tat; in other words, HIV-1C upregulates IDO and downregulates the 5-HTT gene less than HIV-1 clade B, suggesting that HIV-1B and $-1 \mathrm{C}$ Tat proteins may play a differential role in the neuropathogenesis of these infections [2].

\section{Drug abuse \& neuroAIDS}

\section{Alcohol/cannabinoids \& neuroAIDS}

Systems approaches for unraveling mechanisms of chronic $\triangle 9$-THC modulation of SIV infection were discussed by Patricia E Molina of the Department of Physiology, Louisiana State University Health Sciences Center, LA, USA. Molina suggested that drugs of abuse may alter host response to HIV infection, by affecting progression of infection, tissue injury and time to death. Several factors can be involved in this; those pertaining to the host response, as well as those related to the ability of the virus to integrate itself into the host genome. The two most commonly used and abused drugs are the cannabinoids (principal chemical constituents of marijuana) and alcohol. Considerable advances have been made in understanding alcoholinduced injury of hepatic, behavioral and neural substrates. Using integrated systems biology analysis, studies have identified salient cellular and molecular signatures that prevail during infection and their modulation by chronic alcohol and cannabinoid administration in a SIV-infected rhesus macaque model. Findings presented at the symposium suggest that chronic alcohol and cannabinoid administration differentially modulate key interconnected and tissue-specific mechanisms responsible for control of disease progression. Contrasting disease phenotypes result from chronic alcohol and cannabinoid administration to SIV-infected nonhuman primates. It is hypothesized that the contrasting phenotypes provide a unique opportunity for systems biology analysis of the epigenetic, genetic, and proteomic profiles prevailing during SIV infection that are translatable to HIV disease progression [3,4].

\section{Alcohol/tobacco \& neuroAIDS}

The central role of cytochrome P450 2A6 (CYP2A6) in tobacco-mediated oxidative stress in macrophages and astrocytes, as well as the underlying implications for HIV-1 pathogenesis and neuroAIDS, was discussed by Santosh Kumar of the School of Pharmacy, University of Missouri-Kansas City, MO, USA. Kumar showed that tobacco consumption/smoking was highly prevalent in HIV patients. Kumar and his colleagues hypothesized that CYP2A6, which metabolizes nicotine and activates NNK, plays a central role in tobacco/nicotine-mediated oxidative stress, leading to increased HIV-1 replication in monocytes/macrophages and astrocytes. To test this hypothesis, the in vitro HIV-1 model U937 monocyte/macrophage and SVGA (a human astrocytic subclone of SVG cells) cell lines and human primary macrophages were used. These studies showed that CYP2A6 is predominantly expressed in monocytes/macrophages [5] and astrocytes [6]. These tests, carried out in HIV-negative smokers, HIV-positive nonsmokers, HIV-positive smokers and healthy volunteers from Cameroon, Africa, showed a positive correlation between smoking and oxidative stress. Kumar and colleagues are further investigating the role of CYP2A6, oxidative stress and HIV-1 replication in nicotine treatment in HIV-infected primary human monocytes/macrophages.

\section{Cocaine \& neuroAIDS}

Despite the widespread use of antiretroviral therapies, a majority of HIV-1 patients develop HAND, and cocaine abuse can exacerbate these clinical manifestations, as was explained by Sanjay B Maggirwar from the University of Rochester Medical Center, NY, USA (coauthored by Meera V Singh, Donna C Davidson and Servio H Ramirez) [7]. Interaction between monocytes and activated platelets leads to the formation of platelet-monocyte complexes (PMCs), and these monocytes exhibit an enhanced capacity for transendothelial migration [8,9]. Maggirwar explained that cocaine is known to cause platelet activation [10], so the goal was determining whether HIV-positive cocaine users have an even greater increase in the number of PMCs found 
in circulation, as compared with HIV-infected individuals. The results indicated that cocaine use during HIV infection enhances the formation of PMCs. Furthermore, Tat-induced inflammation promotes blood-brain barrier (BBB) permeability in a platelet-dependent manner and increases monocyte adherence to brain microvascular endothelial cells. As cocaine use leads to increased PMCs in HIV-infected individuals, he suggested that this may be a mechanism by which HAND is exacerbated in HIV-positive cocaine users.

The relationship between HIV infection and cocaine abuse was explained by Shilpa Buch from the University of Nebraska Medical Center, Omaha, NE, USA (coauthored by Honghong Yao). The use of illicit drugs worsens the progression of the infection. Buch stated that understanding the mechanism by which HIV and cocaine synergize to enhance the progression of HAND is critical for the development of therapeutic options aimed at targeting disease pathogenesis. Combinatorial cell culture and in vivo experiments were performed to check the effect of HIV neurotoxic protein Tat and/or cocaine on glial cell activation, brain endothelial cell disruption and neuronal toxicity. Protein and mRNA expression of activation markers were used in conjunction with immunostaining, western blotting and chromatin immunoprecipitation assays to understand the changes in gene expression and the signaling pathways involved. The findings suggest that cocaine is a multifactorial agent that uses signaling pathways identical to those used by HIV Tat, thereby affecting activation of glial cells and the permeability of brain endothelial cells, and potentiating the toxicity of neuronal cells [11,12].

\section{METH \& neuroAIDS}

The relation between HIV-1, METH and astrocyte glutamate regulation, which cause combined excitotoxic implications in neuroAIDS, was explained by Anuja Ghorpade from the Department of Cell Biology and Anatomy, University of North Texas Health Science Center, TX, USA (coauthored by Irma L Cisneros). Glutamate is the most abundant excitatory transmitter in the brain, and can lead to neurotoxicity if not properly regulated. Excitotoxicity is a direct result of abnormal regulation of glutamate concentrations in the synapse, and is a common neurotoxic mediator associated with neurodegenerative disorders. It is well accepted that METH, a potent CNS stimulant with high abuse potential, and HIV-1 are implicated in the progression of neurocognitive malfunction.
Both have been shown to induce common neurodegenerative effects, such as astrogliosis, compromised BBB integrity, excitotoxicity and hyperthermia in the brain. However, the cellular and molecular mechanisms of astrocyte-mediated excitotoxicity in the context of METH and HIV-1 are undefined. The results indicated that METH, HIV-1, IL-1 $\beta$ and hyperthermia affect astrocytes and result in the modulation of EAAT2 mRNA and protein expression. To further study METH's mechanism of action in astrocytes, an orphan G-protein-coupled receptor, TAAR1, which binds METH and modulates intracellular cAMP levels, was investigated. Astrocyte TAAR1 activation is induced in a dose-dependent manner by both METH and $\beta$-phenylethylamine, a TAAR1 agonist $[13,14,15]$.

Howard S Fox of University of Nebraska Medical Center (NE, USA) gave an excellent talk on METH and response to viral infection in vivo. Anil Kumar of the University of MissouriKansas City, explained that the synergistic cooperation between METH and HIV-1 gp120 through the P13K/Akt pathway induces IL-6, but not IL-8 expression in astrocytes.

\section{Morphine \& neuroAIDS}

Morphine induction of Toll-like receptors (TLRs) on glial cells, which potentiates HIV-1induced neuropathogenesis, was explained by Sabita Roy from the University of Minnesota, MN, USA (coauthored by Raini Dutta and Anitha G Krishnan). There is a strong correlation between chronic opioid use and HIV infection. Progression to AIDS dementia/HAND is markedly accelerated in opiate drug abusers $[16,17,18]$. Clinically, microglial activation better correlates with HAND than productive HIV-1 infection in the CNS. It was explained that coinfection with Streptococcus pneumoniae (S.p) contributes to the increased prevalence of HAND in the opioid drug-abusing population. The role of TLRs in morphine modulation of proinflammatory cytokines is important, as significant increase in TLR expression has been observed following morphine use, while TAT and S.p increase TLR activation, resulting in a threefold, synergistic increase in proinflammatory cytokine (IL-6 and TNF- $\alpha$ ) levels with concurrent increase in microglial, reactive oxidative species and nitric oxide, suggesting that activation of TLRs on microglial cells by morphine and Tat, in the context of S.p infection, may be a potential mechanism for the increased prevalence of HAND in HIV-infected opioid drug users. 
Mechanisms underlying morphine-induced conditioned place preference in the HIV-1 transgenic rat were explained by Sulie L Chang of the Institute of NeuroImmune Pharmacology $\&$ Department of Biological Sciences, Seton Hall University, NY, USA. A bidirectional interaction exists between the central nervous and immune systems, which was discovered by identifying the converging actions of substances of abuse in altering the neuronal and immune pathways [19]. Opiates also accelerate the progression of sepsis caused by the bacterial endotoxins, lipopolysaccharides (LPS), to septic shock [20]. Antiretroviral therapies such as HAART have been successful in limiting viral load and maintaining a relatively healthy immune response; however, HIV-1 viral proteins are still expressed.

HIV-1-infected individuals have a higher probability of abusing addictive substances [21]. Using HIV-1Tg rats, a noninfectious rodent model for patients on HAART, it was found that exposure to LPS increases cytokine levels in the rat brain, and that HIV-1Tg rats are more responsive to substances of abuse [22]. LPS increases expression of the $\mu$-opioid receptor to a greater extent in HIV-1Tg rats than in normal rats, indicating that there may be a synergistic effect between HIV-1 infection and bacterial infections that renders HIV-1-infected individuals more susceptible to morphine dependence. Memantine, an Alzheimer's medication, decreases LPS-induced microglial activation, possibly by reducing the production of inflammatory cytokines and partially decreasing morphine-induced conditioned place preference via its anti-inflammatory effects [23].

\section{Treatment}

A mechanism for multifunctional nanocarrier drug delivery, which can be helpful in treating neuroAIDS and opiate addicts, was explained by Madhavan PN Nair (coauthored by Sakhrat Khizroev and Shailendra K Saxena). Nair's presentation began with the idea that HIV-1 proteins and opiates perform in synergy to potentiate HIV-related neurotoxicity, and that this neurotoxicity results in neuroAIDS development. The current lack of effective treatment against neuroAIDS is due to the impermeability of the $\mathrm{BBB}$, which prevents most drug molecules from crossing it. Nair highlighted the novel concept of a magnetic nanocarrier which, under the influence of an external magnetic field, can be potent in delivering AZT 5'-triphosphate (the active form of AZT) and CTOP (a potent $\mu$-opioid receptor antagonist) [24,25] into the brain to alleviate neuroAIDS and opiate addiction.

The presentations and posters from young investigators explaining possible mechanisms of different drugs of abuse in HIV and neuroAIDS, including treatment and therapeutic strategies, were very impressive. Overall, it is important to understand the deep underlying mechanisms and alterations that finally lead to the chain of reactions that result in neuroAIDS. It should also be kept in mind - and explained to the public that the depression that is part of neuroAIDS is associated with the increasing viral load in the patient's brain and attacks on the CNS cells, and not only with social stigma and related issues.

\section{Conclusion \& future directions}

Finally, Jag H Khalsa emphasized that with an expanding neuroAIDS patient population and increasing drug abuse worldwide, it is important to understand the mechanisms of neurological complications and HIV disease progression in the brain in order to design appropriate prevention/treatment strategies, while Jeymohan Joseph stressed the importance of viral and host genetics in HIV neuropathogenesis. HIV enters the CNS at an early point following infection, subsequently infecting all CNS cells, leading to HIV encephalitis and dementia. Additionally, in cases where infected individuals are drug users, the nervous system is also a target for drugs of abuse, which can lead to ischemic lesions secondary to the inflammation of medium and small vessels [26] and abnormal metabolism in brain tissue, even in subjects who are otherwise asymptomatic [27]. Viral effects of HIV on the CNS is a novel field that is attracting scientists ranging from epidemiology of neuroAIDS to molecular and genetic bases of neuroAIDS, the role of recreational drugs on HIV infection and immune responses, differential neuropathology in HIV clades to the use of nanotechnology for drug delivery to treat neurological complications of HIV.

The symposium clearly pointed out the need for further research on several themes that could be targeted for future research. These could include, but not be limited to, the study of the underlying molecular/genetic/epigenetic bases of neuropathology/encephalopathy in HIV-infection, the role of recreational drugs on differential neuropathogenesis induced by HIV clades, the expression of immune system genes that confer specific susceptibility to HIV infection, in vitro models that closely 
mimic conditions seen with natural infection, mechanisms that confer long-term protection to HIV-infected individuals on brain metabolism and the effect of drug usage and opportunistic infections, developing innovative interventions for the management of neuroAIDS and associated consequences, training additional researchers/clinicians in the field of neuropsychiatric complications and developing neuropathology facilities.

\begin{abstract}
Acknowledgments
The authors are grateful to the delegates for sharing their abstracts for this article. We sincerely thank the Director, CCMB (CSIR) $C M$ Rao, and Founder-Managing Director of OMICS Group Inc., USA, SB Gedela and his team for the hosting, encouragement and support for the Symposium on Drugs of Abuse - HIV Infection and NeuroAIDS: A Global Perspective, during the 3rd World Congress on Biotechnology at HICC, Hyderabad, India.
\end{abstract}

\section{Financial \& competing interests disclosure}

The authors are grateful to Council of Scientific and Industrial Research (CSIR-CCMB), India, for the encouragement and support for this work. SK Saxena and MPN Nair are supported by NIH grants R37DA025576 and R01MH085259. The authors have no other relevant affiliations or financial involvement with any organization or entity with a financial interest in or financial conflict with the subject matter or materials discussed in the manuscript apart from those disclosed.

No writing assistance was utilized in the production of this manuscript.

\section{References}

1. Saxena SK, Tiwari S, Nair MPN. A global perspective on HIV/AIDS. Science 337(6096), 798 (2012).

2. Samikkannu T, Rao KV, Gandhi N et al. Human immunodeficiency virus type 1 clade $\mathrm{B}$ and $\mathrm{C}$ Tat differentially induce indoleamine 2,3-dioxygenase and serotonin in immature dendritic cells: implications for neuroAIDS. J. Neurovirol. 16(4), 255-263 (2010).

3. Molina PE, Amedee A, LeCapitaine NJ et al. Cannabinoid neuroimmune modulation of SIV disease. J. Neuroimmune Pharmacol. 6(4), 516-527 (2011)

4. Molina PE, Lang $\mathrm{CH}, \mathrm{McNurlan} \mathrm{M}$ et al. Chronic alcohol accentuates simian acquired immunodeficiency syndrome-associated wasting. Alcohol Clin. Exp. Res. 32(1), 138-147 (2008).

5. Jin M, Earla R, Shah A et al. A LC-MS/MS method for concurrent determination of
CYP2A6-mediated nicotine metabolites and analysis of nicotine-mediated oxidative stress in U937 macrophages. J. Neuroimmune Pharmacol. 7(1), 289-299 (2012).

6. Ande A, Earla R, Jin M et al. An LC-MS/ MS method for concurrent determination of nicotine metabolites and the role of CYP2A6 in nicotine metabolite-mediated oxidative stress in SVGA astrocytes. Drug Alcohol Depend. 125(1-2), 49-59 (2012).

7. Heaton RK, Clifford DB, Franklin DR et al. HIV-associated neurocognitive disorders persist in the era of potent antiretroviral therapy: CHARTER Study. Neurology 75(23), 2087-2096 (2010).

8. Singh MV, Davidson DC, Kiebala M et al. Detection of circulating platelet-monocyte complexes in persons infected with human immunodeficiency virus type-1. J. Virol. Methods 181(2), 170-176 (2012).

9. Huo Y, Schober A, Forlow SB et al. Circulating activated platelets exacerbate atherosclerosis in mice deficient in apolipoprotein E. Nat. Med. 9(1), 61-67 (2003).

10. Heesch CM, Wilhelm CR, Ristich J et al. Cocaine activates platelets and increases the formation of circulating platelet containing microaggregates in humans. Heart 83(6), 688-695 (2000).

11. Yao H, Duan M and Buch S. Cocainemediated induction of platelet-derived growth factor: implication for increased vascular permeability. Blood 117(8), 2538-2547 (2011).

12. Yao H, Kim K, Duan M et al. Cocaine hijacks sigma-1 receptor to initiate induction of ALCAM: implication for increased monocyte adhesion and migration in the central nervous system. J. Neuroscience 31(16), 5942-5955 (2011).

13. Kass MD, Liu X, Vigorito M et al. Methamphetamine-induced behavioral and physiological effects in adolescent and adult HIV-1 transgenic rats. J. Neuroimmune Pharmacol. 5(4), 566-573 (2010).

14. Kiyatkin EA, Sharma HS. Acute methamphetamine intoxication: brain hyperthermia, blood-brain barrier, brain edema, and morphological cell abnormalities. Int. Rev. Neurobiol. 88, 65-100 (2009).

15. Revel FG, Moreau JL, Gainetdinov RR et al. TAAR1 activation modulates monoaminergic neurotransmission, preventing hyperdopaminergic and hypoglutamatergic activity. Proc. Natl Acad. Sci. USA 108(20), 8485-8490 (2011).

16. Nath A. Human immunodeficiency virus (HIV) proteins in neuropathogenesis of HIV dementia. J. Infect. Dis. 186(2), S193-S198 (2002).

17. Roy S, Ninkovic J, Banerjee S et al. Opioid drug abuse and modulation of immune function: consequences in the susceptibility to opportunistic infections. J. Neuroimmune Pharmacol. 6(4), 442-465 (2011).

18. Dutta R, Roy S. Mechanism(s) involved in opioid drug abuse modulation of HAND. Curr. HIV Res. 10(5), 469-477 (2012).

19. Chang SL, Patel NA, Romero AA. Activation and desensitization of Fos immunoreactivity in the rat brain following ethanol administration. Brain Res. 679, 89-98 (1995).

20. Ocasio FM, Jiang Y, House SD et al. Chronic morphine accelerates the progression of lipopolysaccharide-induced sepsis to septic shock. J. Neurol. 149, 90-100 (2004).

21. Chang SL, Beltran JA, Swarup S. Expression of the mu opioid receptor in the human immunodeficiency virus type 1 transgenic rat model. J. Virol. 81(16), 8406-8411 (2007).

22. Chang SL, Connaghan KP. Behavioral and molecular evidence for a feedback interaction between morphine and HIV-1 viral proteins. J. Neuroimmune Pharmacol. 7(2), 332-340 (2012).

23. Chen SL, Tao PL, Chu CH et al. Low-dose memantine attenuated morphine addictive behavior through its antiinflammation and neurotrophic effects in rats. J. Neuroimmune Pharmacol. 7(2), 444-453 (2012).

24. Saiyed ZM, Gandhi NH, Nair MP. AZT 5'-triphosphate nanoformulation suppresses Human immunodeficiency virus type 1 replication in peripheral blood mononuclear cells. J. Neurovirol. 15(4), 343-347 (2009).

25. Saiyed ZM, Gandhi NH, Nair MPN. Magnetic nanoformulation of Azidothymidine 5'-triphosphate for targeted delivery across the blood-brain barrier. Int J. Nanomed. 5, 157-166 (2010). 
26. Anderson E, Zink W, Xiong $\mathrm{H}$ et al. HIV-1-associated dementia: a metabolic encephalopathy perpetrated by virus-infected and immune-competent mononuclear phagocytes. J. Acquir. Immune Defic. Syndr. 31(Suppl. 2), S43-S54 (2002).

27. Chang L, Ernst T, Strickland T et al. Gender effects on persistent cerebral metabolite changes in the frontal lobes of abstinent cocaine users. Am. J. Psychiatry 156(5), 716-722 (1999).

\section{Websites}

101. United Nations Office of Drugs and Crime (UNODC), World Drug Report 2012

(United Nations publication, sales no. E.12. XI.1) 2012. www.unodc.org/documents/data-andanalysis/WDR2012/WDR_2012_web_ small.pdf

(Accessed 1 November 2012)

102. Department of Health and Human Services. http:/grants.nih.gov/grants/guide/pa-files/ PA-11-014.html

(Accessed 1 November 2012) 